Tropical Journal of Pharmaceutical Research June 2016; 15 (6): 1267-1274

ISSN: $1596-5996$ (print); 1596-9827 (electronic) (C) Pharmacotherapy Group, Faculty of Pharmacy, University of Benin, Benin City, 300001 Nigeria.

\title{
Synthesis and antibacterial study of some s-substituted aliphatic analogues of 2-mercapto-5-(1-(4-toluenesulfonyl) piperidin-4-yl)-1,3,4-oxadiazole
}

\author{
A Sattar ${ }^{1}$, Aziz-ur-Rehman ${ }^{1 *}$, MA Abbasi $^{1}$, SZ Siddiqi $^{1}$, K Nafeesa $^{1}$ and I Ahmad ${ }^{2}$ \\ ${ }^{1}$ Department of Chemistry, Government College University, Lahore-54000, ${ }^{2}$ Department of Biochemistry and Biotechnology, \\ The Islamia University of Bahawalpur, Bahawalpur-63100, Pakistan
}

*For correspondence: Email: azizryk@yahoo.com, rehman@gcu.edu.pk; Tel: (+92)-42-111000010

Received: 17 October 2015

Revised accepted: 12 May 2016

\begin{abstract}
Purpose: To synthesize a series of analogues of 1,3,4-oxadiazole and to evaluate their antibacterial activity.

Methods: Ethyl piperidin-4-carboxylate (1) was mixed with 4-toluenesulfonyl chloride (2) in benignant conditions to yield ethyl 1-(4-toluenesulfonyl)piperidin-4-carboxylate (3) and then 1-(4toluenesulfonyl)piperidin-4-carbohydrazide (4). Intermolecular cyclization of 4 into 2-mercapto-5-(1-(4toluenesulfonyl) piperidin-4-yl)-1,3,4-oxadiazole (5) was obtained on reflux with CS2 in the presence of $\mathrm{KOH}$. Molecule 5 was stirred with alkyl halides, 6a-i, in DMF in the presence of LiH to synthesize the final compounds, 7a-i. The structures of these molecules were elucidated by Fourier transform infra-red (FTIR) spectroscopy, proton nuclear magnetic resonance $\left({ }^{1} \mathrm{H}-\mathrm{NMR}\right)$ and electron impact mass spectrometry (EI-MS). Antibacterial activity was evaluated against five bacterial strains, namely, Salmonella typhi, Escherichia coli, Pseudomonas aeruginosa, Staphylococcus aureus and Bacillus subtilis, with ciprofloxacin used as standard antibacterial agent.

Results: Out of nine synthesized derivatives, compound 7a was the most active against three bacterial strains, S. typhi, E. coli and P. aeruginosa, with minimum inhibitory concentration (MIC) of $9.11 \pm 0.40$, $9.89 \pm 0.45$ and $9.14 \pm 0.72 \mu \mathrm{M}$, respectively, compared with $7.45 \pm 0.58,7.16 \pm 0.58$ and $7.14 \pm 0.18$ $\mu M$, respectively, for the reference standard (ciprofloxacin). Similarly, compounds 7a - 7c showed relatively good antibacterial activity against $B$. subtilis strain while compound $7 e-7 g$ revealed good results against $S$. typhi bacterial strain.

Conclusion: The results indicate that S-substituted derivatives of the parent compound are more effective antibacterial agents than the parent compound, even with minor differences in substituents.
\end{abstract}

Keywords: 1,3,4-Oxadiazole, Antibacterial activity, Ethyl piperidin-4-carboxylate, Sulfonamide

Tropical Journal of Pharmaceutical Research is indexed by Science Citation Index (SciSearch), Scopus, International Pharmaceutical Abstract, Chemical Abstracts, Embase, Index Copernicus, EBSCO, African Index Medicus, JournalSeek, Journal Citation Reports/Science Edition, Directory of Open Access Journals (DOAJ), African Journal Online, Bioline International, Open-J-Gate and Pharmacy Abstracts

\section{INTRODUCTION}

Derivatives of 1,3,4-oxadiazole, aromatic heterocyclic class bearing two nitrogens and one oxygen, have demonstrated a broad spectrum of biological activities including anticonvulsant, antidepressant, anti-inflammatory, antifungal, hypoglycemic, antimitotic, antimicrobial, analgesic, insecticidal, herbicidal and anticancer activities [1-11]. Piperidine, derivatives are known for the therapeutic potential [12]. They have been employed to control insulin \& glucose levels, cocaine abuse treatment, and as anesthetics [13]. The sulfamoyl bearing molecules, sulfonamides, have been used as antibacterial agents, anti-enzymatic agents to 
cure different enzyme relating diseases, in chemotherapy of cancer, diuretics and hypoglycemia [14-16].

Scientists have been searching for new drug candidate for new infectious diseases of humans, plants and animals. In this field the derivatives of sulfonamide, oxadiazole especially 1,3,4-isomer and piperidine have earned much fame because of significant pharmacological activities, as referenced. Their biological activities prompted us to synthesize some new compounds of 1,3,4oxadiazole, piperidine and sulfonamide moieties after multi-steps and to evaluate them for antibacterial activity against two gram positive and three gram negative bacterial strains.

\section{EXPERIMENTAL}

\section{General}

The synthetic grade chemicals were purchased from Sigma Aldrich, Alfa Aesar and Merck through local suppliers. The solvents were of analytical grade and used without purification. Single spot on pre-coated silica gel G-25-UV254 aluminum plates in thin layer chromatography (TLC) was the initial tool for purity of compounds during and after the reactions. The TLC plates were developed in solvent system of ethyl acetate and n-hexane and observed under 254 $\mathrm{nm}$ UV lamps. Griffin-George melting point apparatus was used to note the melting points using open capillary tube and were uncorrected. Jasco-320-A spectrometer was employed to record the IR spectra using $\mathrm{KBr}$ pellet method and wave number was mentioned in $\mathrm{cm}^{-1}$. Bruker spectrometer operating at $400 \mathrm{MHz}$ was utilized to record the proton NMR spectra of the compounds dissolved in $\mathrm{CDCl}_{3}$ and chemical shifts were given in ppm. JMS-HX-110 spectrometer was applied to record the EIMS spectra.

\section{Synthesis of ethyl 1-(4-toluenesulfonyl) piperidin-4-carboxylate (3)}

Ethyl piperidin-4-carboxylate $(1 ; 15 \mathrm{mmol})$ was taken in $100 \mathrm{~mL}$ round bottom (RB) flask and suspended in 25 - $30 \mathrm{~mL}$ distilled water. 4Toluenesulfonyl chloride (2; $15 \mathrm{mmol})$ was gradually added by parts on continuous stirring. The mixture was allowed to stir for further $4 \mathrm{~h}$ and the $\mathrm{pH}$ was adjusted to $9-10$ by aqueous $\mathrm{Na}_{2} \mathrm{CO}_{3}$ at room temperature (RT). On completion of reaction to quench the precipitates a few drops of dilute $\mathrm{HCl}$ were added to the reaction mixture on continuous shaking till $\mathrm{pH}$ of 5-6 was adjusted. The precipitates were allowed to settle down for $0.25 \mathrm{~h}$. The precipitates were filtered, washed with distilled water and dried.

\section{Synthesis of 1-(4-toluenesulfonyl)piperidin-4- carbohydrazide (4)}

Ethyl 1-(4-toluenesulfonyl) piperidin-4-carboxylate $(3 ; 12 \mathrm{mmol})$ was homogeneously mixed with $25 \mathrm{~mL} \mathrm{MeOH}$ in a $100 \mathrm{~mL}$ RB flask. The hydrated hydrazine $(80 \%, 12 \mathrm{mmol})$ was poured to the mixture and set to reflux for $5 \mathrm{~h}$. After single spot on TLC, excess of distilled water was added along with shaking till precipitation. The product was separated through filtration, washed with distilled water and dried.

\section{Synthesis of 2-mercapto-5-(1-(4-toluenesul- fonyl)piperidin-4-yl)-1,3,4-oxadiazole (5)}

Compound 4 (10 mmol) was taken in $100 \mathrm{~mL} \mathrm{RB}$ flask and mixed with $20 \mathrm{~mL}$ methanol. Then 10 mmol $\mathrm{KOH}$ was dissolved in the mixture on reflux. The system was cooled to RT and 20 mmol CS2 was added and again set to reflux for 7 h. After confirmation by TLC, excess cold distilled water (about $40 \mathrm{~mL}$ ) was added followed by dilute $\mathrm{HCl}$ to adjust $\mathrm{pH}$ at 5-6 and left the precipitates to settle down for $0.25 \mathrm{~h}$. The precipitates of $\mathbf{5}$ were collected after filtration, washed with distilled water and dried.

\section{Synthesis of 2-alkylthio-5-(1-(4- toluenesulfonyl)piperidin-4-yl)-1,3,4- oxadiazole (7a-i)}

The molecule $5(0.2 \mathrm{mmol})$ was taken in $50 \mathrm{~mL}$ RB flask and dissolved in N, Ndimethylformamide (DMF, $12 \mathrm{~mL}$ ). Lithium hydride $(0.2 \mathrm{mmol})$ was added and the mixture was stirred for $0.5 \mathrm{~h}$ at RT. Then alkyl halides $(6 a-i, 0.2 \mathrm{mmol})$ were added and set to stir for 4$6 \mathrm{~h}$. On completion of reaction, confirmed by TLC, excess chilled distilled water was added to gain the precipitates of final products, $7 a-i$, which were collected after filtration from aqueous medium, washed, dried and then subjected to antibacterial activity analysis.

\section{Antibacterial activity assay}

Two gram-positive bacterial strains and three gram-negative bacterial strains were clinically isolated and stored on agar medium to facilitate the bacterial growth. $20 \mu \mathrm{g}$ test samples after dilution by suited solvents and $180 \mu \mathrm{L}$ overnight maintained fresh bacterial cultures after suited dilution with fresh nutrient broth were mixed. Initial absorbance was observed and kept between $0.12-0.19$ at $540 \mathrm{~nm}$. 


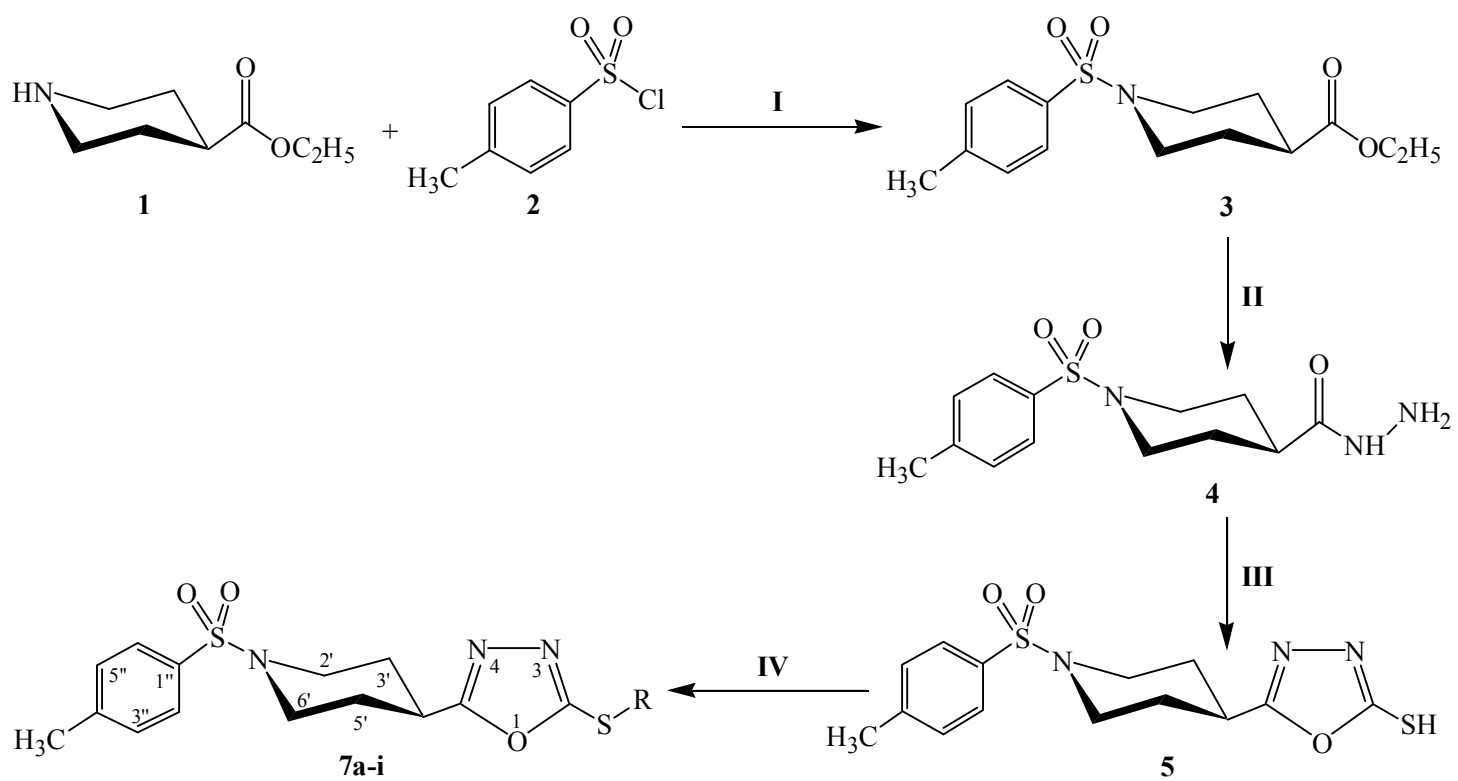

Scheme-1: Protocol for S-substituted derivatives of 2-mercapto-5-(1-(4-toluenesulfonyl) piperidin-4-yl)-1,3,4oxadiazole (5). Reagents and conditions: (I) water, sodium carbonate, stirring for $4 \mathrm{~h}$ (II) hydrated hydrazine, methanol, refluxing for $5 \mathrm{~h}$ (III) carbon disulfide, potasium hydroxide, methanol, refluxing for $7 \mathrm{~h}$ (IV) alkyl halides (6a-i), LiH, DMF, Stirred for $4-6 \mathrm{~h}$.

Table 1: Different S-substituted alkyl groups

\begin{tabular}{|c|c|c|c|}
\hline Compound & $\mathbf{R}$ & Compound & $\mathbf{R}$ \\
\hline $7 a$ & $-\mathrm{CH}_{2}-\mathrm{CH}_{3}$ & $7 f$ & $-\mathrm{CH}_{2}-\mathrm{CH}_{2}-\mathrm{CH}_{2}-\mathrm{CH}_{2}-\mathrm{CH}_{3}$ \\
\hline $1 \mathrm{a}$ & $1 " ' \quad 2 " '$ & IT & $\begin{array}{lllll}1 " \prime \prime & 2 " \prime & 3{ }^{\prime \prime \prime} & 4{ }^{\prime \prime \prime} & 5^{\prime \prime \prime}\end{array}$ \\
\hline $7 b$ & 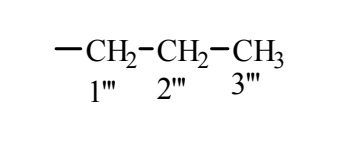 & $7 g$ & 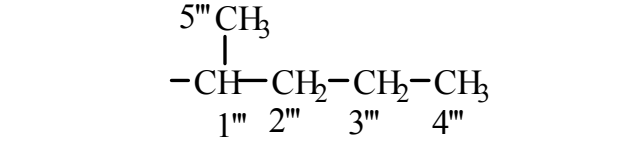 \\
\hline $7 c$ & $\underset{3^{\prime \prime \prime}}{\mathrm{CH}_{3}}$ & $7 \mathrm{~h}$ & 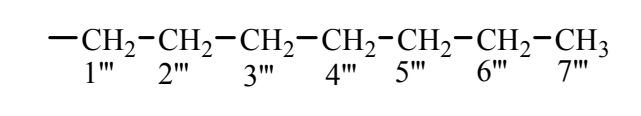 \\
\hline $7 d$ & 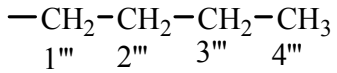 & $7 i$ & 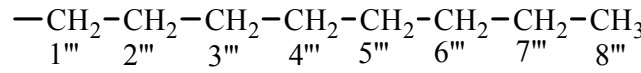 \\
\hline $7 e$ & $\begin{array}{c}-\mathrm{C} H-\mathrm{CH}_{2}-\mathrm{CH}_{3} \\
1^{\prime \prime \prime} \quad 2^{\prime \prime \prime} \quad 3^{\prime \prime \prime}\end{array}$ & & \\
\hline
\end{tabular}

Microplate with lid was incubated at $37{ }^{\circ} \mathrm{C}$ for 16 - $24 \mathrm{~h}$. Absorbance was noted at $540 \mathrm{~nm}$ using micro plate reader before and after incubation and the difference was noted as an index of bacterial growth. The percent inhibition was calculated using Eq 1.

Inhibition $(\%)=(\mathrm{C}-\mathrm{T}) / \mathrm{C} \times 100$

where $\mathrm{C}$ and $\mathrm{T}$ are absorbance of control and test compound, respectively.

Results are mean of triplicate measurements ( $\mathrm{n}$ $=3, \pm$ SEM). Ciprofloxacin was taken as standard. Minimum inhibitory concentration (MIC) was measured with suitable dilutions (5 - 30 $\mu \mathrm{g} /$ well) and results were calculated using EZ-Fit software (Perrella Scientific Inc. Amherst USA) [17].

\section{Statistical analysis}

All computations were executed in triplicate and statistical analysis was performed with Microsoft Excel 2010. The results are expressed as mean \pm SEM $(n=3)$. Minimum inhibitory concentration (MIC) was computed with suitable dilutions (5 $30 \mu \mathrm{g} /$ well) for each sample and results calculated using EZ-Fit software (Perrella Scientific Inc, Amherst, USA). 


\section{RESULTS}

Multifunctional compound $\mathbf{5}$ was synthesized successfully, this compound contained 1,3,4oxadiazole, sulfamoyl and piperidine moieties. The synthesis of the compound is represented in Scheme-1. Different S-substituted derivatives of compound $\mathbf{5}$ were synthesized in good yield. All these derivatives were screened for their antibacterial activity. The antibacterial studies proved compound $\mathbf{7 a}$ the most potent of all compounds under study while some other compounds showed relatively good antibacterial potential against different bacterial strains.

\section{Ethyl 1-(4-toluenesulfonyl) piperidin-4- carboxylate (3)}

White amorphous solid; Yield: $89 \%$; M.P.: 70-72 ${ }^{\circ} \mathrm{C}$; Mol. formula: $\mathrm{C}_{15} \mathrm{H}_{21} \mathrm{NO}_{4} \mathrm{~S}$; Mol. weight: 311; IR $\left(\mathrm{KBr}, \mathrm{cm}^{-1}\right): U_{\max } 3067$ (Aromatic C-H), 1732 (Ester $\mathrm{C}=\mathrm{O}), 1531 \quad$ (Aromatic $\mathrm{C}=\mathrm{C}), 1335$ (Sulfamoyl $\mathrm{S}=\mathrm{O}$ ), 1079 (Ester C-O); ${ }^{1} \mathrm{H}-\mathrm{NMR}$ (400 MHz, $\left.\mathrm{CDCl}_{3}, \mathrm{ppm}\right): \delta 7.62(\mathrm{~d}, J=8.0 \mathrm{~Hz}$, $2 \mathrm{H}, \mathrm{H}-2$ " \& H-6"), 7.32 (d, J = 8.0 Hz, 2H, H-3" \& $\mathrm{H}-5^{\prime \prime}$ ), 3.98 (q, J = 7.2 Hz, 2H, OCH $\mathrm{CH}_{3}$ ), 3.71$3.68\left(\mathrm{~m}, 2 \mathrm{H}, \mathrm{H}_{\mathrm{e}}-2^{\prime} \& \mathrm{H}_{\mathrm{e}}-6^{\prime}\right), 2.73-2.62(\mathrm{~m}, 1 \mathrm{H}, \mathrm{H}-$ 4'), 2.54-2.48 (m, $\left.2 \mathrm{H}, \mathrm{H}_{\mathrm{a}}-2^{\prime} \& \mathrm{H}_{\mathrm{a}}-6^{\prime}\right), 2.42$ (s, 3H, $\left.\mathrm{CH}_{3}-4^{\prime \prime}\right), 2.10-2.08\left(\mathrm{~m}, 2 \mathrm{H}, \mathrm{H}_{\mathrm{e}}-3^{\prime} \& \mathrm{H}_{\mathrm{e}}-5^{\prime}\right), 1.60-$ $1.86\left(\mathrm{~m}, 2 \mathrm{H}, \mathrm{H}_{\mathrm{a}}-3^{\prime} \& \mathrm{H}_{\mathrm{a}}-5^{\prime}\right), 1.15(\mathrm{t}, \mathrm{J}=7.2 \mathrm{~Hz}$, $\left.\mathrm{OCH}_{2} \mathrm{CH}_{3}\right) ; \quad \operatorname{EIMS}(\mathrm{m} / \mathrm{z}): 311[\mathrm{M}]^{+}, \quad 266$ $\left[\mathrm{C}_{13} \mathrm{H}_{16} \mathrm{NO}_{3} \mathrm{~S}\right]^{+}, \quad 238 \quad\left[\mathrm{C}_{12} \mathrm{H}_{16} \mathrm{NO}_{2} \mathrm{~S}\right]^{+}, \quad 184$ $\left[\mathrm{C}_{8} \mathrm{H}_{10} \mathrm{NO}_{2} \mathrm{~S}\right]^{+}, 170\left[\mathrm{C}_{7} \mathrm{H}_{8} \mathrm{NO}_{2} \mathrm{~S}\right]^{+}, 155\left[\mathrm{C}_{7} \mathrm{H}_{7} \mathrm{O}_{2} \mathrm{~S}\right]^{+}$, $91\left[\mathrm{C}_{7} \mathrm{H}_{7}\right]^{+}$.

\section{1-(4-Toluenesulfonyl) piperidin-4-carbohy- drazide (4)}

White crystalline solid; Yield: $91 \%$; M.P.: 128$130{ }^{\circ} \mathrm{C}$; Mol. formula: $\mathrm{C}_{13} \mathrm{H}_{19} \mathrm{~N}_{3} \mathrm{O}_{3} \mathrm{~S}$; Mol. weight: 297; IR ( $\left.\mathrm{KBr}, \mathrm{cm}^{-1}\right): U_{\max } 3348$ (Hydrazide $\mathrm{N}-\mathrm{H}$ ), 3063 (Aromatic C-H), 1682 (Amide $\mathrm{C}=\mathrm{O}$ ), 1534 (Aromatic $\mathrm{C}=\mathrm{C}$ ), 1339 (Sulfamoyl $\mathrm{S}=\mathrm{O}$ ); ${ }^{1} \mathrm{H}-\mathrm{NMR}$ $\left(400 \mathrm{MHz}, \mathrm{CDCl}_{3}, \mathrm{ppm}\right): \delta 7.61(\mathrm{~d}, J=8.0 \mathrm{~Hz}$, $2 \mathrm{H}, \mathrm{H}-2$ " \& H-6"), 7.33 (d, J = $8.0 \mathrm{~Hz}, 2 \mathrm{H}, \mathrm{H}-3$ " \& $\left.\mathrm{H}-5^{\prime \prime}\right), 3.72-3.69\left(\mathrm{~m}, 2 \mathrm{H}, \mathrm{H}_{\mathrm{e}}-2^{\prime} \& \mathrm{H}_{e}-6^{\prime}\right), 2.73-2.62$ (m, 1H, H-4'), 2.53-2.49 (m, 2H, $\left.\mathrm{H}_{a}-2^{\prime} \& \mathrm{H}_{a}-6^{\prime}\right)$, 2.42 (s, 3H, $\left.\mathrm{CH}_{3}-4 "\right), 2.12-2.10\left(\mathrm{~m}, 2 \mathrm{H}, \mathrm{H}_{e}-3^{\prime}\right.$ \& $\left.\mathrm{H}_{e}-5^{\prime}\right)$, 1.58-1.84 (m, 2H, $\left.\mathrm{H}_{a}-3^{\prime} \& \mathrm{H}_{a}-5^{\prime}\right)$; EIMS $(\mathrm{m} / \mathrm{z}): 297 \quad[\mathrm{M}]^{+}, \quad 266 \quad\left[\mathrm{C}_{13} \mathrm{H}_{16} \mathrm{NO}_{3} \mathrm{~S}\right]^{+}, \quad 238$ $\left[\mathrm{C}_{12} \mathrm{H}_{16} \mathrm{NO}_{2} \mathrm{~S}\right]^{+}, \quad 184 \quad\left[\mathrm{C}_{8} \mathrm{H}_{10} \mathrm{NO}_{2} \mathrm{~S}\right]^{+}, \quad 170$ $\left[\mathrm{C}_{7} \mathrm{H}_{8} \mathrm{NO}_{2} \mathrm{~S}\right]^{+}, 155\left[\mathrm{C}_{7} \mathrm{H}_{7} \mathrm{O}_{2} \mathrm{~S}\right]^{+}, 91\left[\mathrm{C}_{7} \mathrm{H}_{7}\right]^{+}$.

\section{2-Mercapto-5-(1-(4-toluenesulfonyl)piperidin- 4-yl)-1,3,4-oxadiazole (5)}

White amorphous solid; Yield: 87 \%; M.P.: 230$233{ }^{\circ} \mathrm{C}$; Mol. formula: $\mathrm{C}_{14} \mathrm{H}_{17} \mathrm{~N}_{3} \mathrm{O}_{3} \mathrm{~S}_{2}$; Mol. weight: 339; IR ( $\left.\mathrm{KBr}, \mathrm{cm}^{-1}\right): U_{\max } 3067$ (Aromatic C-H),
2522 (Mercapto S-H), 1641 (Oxadiazole C=N), 1541 (Aromatic $\mathrm{C}=\mathrm{C}$ ), 1345 (Sulfamoyl $\mathrm{S}=\mathrm{O}$ ), 1249 \& 1079 (Oxadiazole C-O-C); ${ }^{1} \mathrm{H}-\mathrm{NMR}(400$ $\left.\mathrm{MHz}, \mathrm{CDCl}_{3}, \mathrm{ppm}\right): \delta 7.63(\mathrm{~d}, J=8.0 \mathrm{~Hz}, 2 \mathrm{H}, \mathrm{H}-$ 2" \& H-6"), 7.32 (d, J = 8.0 Hz, 2H, H-3" \& H-5"), 3.71-3.68 (m, 2H, $\left.\mathrm{H}_{e}-2^{\prime} \& \mathrm{H}_{e}-6^{\prime}\right)$, 2.74-2.63 (m, $\left.1 \mathrm{H}, \mathrm{H}-4^{\prime}\right), 2.54-2.48\left(\mathrm{~m}, 2 \mathrm{H}, \mathrm{H}_{\mathrm{a}}-2^{\prime} \& \mathrm{H}_{a}-6^{\prime}\right), 2.42$ (s, 3H, $\left.\mathrm{CH}_{3}-4^{\prime \prime}\right), 2.10-2.08\left(\mathrm{~m}, 2 \mathrm{H}, \mathrm{H}_{e}-3^{\prime} \& \mathrm{H}_{e}-5^{\prime}\right)$, 1.59-1.85 (m, 2H, $\left.\mathrm{H}_{a}-3^{\prime} \& \mathrm{H}_{a}-5^{\prime}\right)$; EIMS (m/z): 339 $[\mathrm{M}]^{+}, 266\left[\mathrm{C}_{13} \mathrm{H}_{16} \mathrm{NO}_{3} \mathrm{~S}\right]^{+}, 238\left[\mathrm{C}_{12} \mathrm{H}_{16} \mathrm{NO}_{2} \mathrm{~S}\right]^{+}, 184$ $\left[\mathrm{C}_{8} \mathrm{H}_{10} \mathrm{NO}_{2} \mathrm{~S}\right]^{+}, 170\left[\mathrm{C}_{7} \mathrm{H}_{8} \mathrm{NO}_{2} \mathrm{~S}\right]^{+}, 155\left[\mathrm{C}_{7} \mathrm{H}_{7} \mathrm{O}_{2} \mathrm{~S}\right]^{+}$, $91\left[\mathrm{C}_{7} \mathrm{H}_{7}\right]^{+}$.

\section{2-Ethylthio-5-(1-(4-toluenesulfonyl)piperidin- 4-yl)-1,3,4-oxadiazole (7a)}

Fluffy white amorphous solid; Yield: $87 \%$; M.P.: 184-186 ${ }^{\circ} \mathrm{C}$; Mol. formula: $\mathrm{C}_{16} \mathrm{H}_{21} \mathrm{~N}_{3} \mathrm{O}_{3} \mathrm{~S}_{2}$; Mol. weight: 367 ; IR $\left(\mathrm{KBr}, \mathrm{cm}^{-1}\right)$ : $U_{\max } 3065$ (Aromatic $\mathrm{C}-\mathrm{H}$ ), 1645 (Oxadiazole $\mathrm{C}=\mathrm{N}$ ), 1563(Aromatic $\mathrm{C}=\mathrm{C}$ ), 1335 (Sulfamoyl S=O), 1249 \& 1079 (Oxadiazole C-O-C); ${ }^{1} \mathrm{H}-\mathrm{NMR} \quad(400 \mathrm{MHz}$, $\left.\mathrm{CDCl}_{3}, \mathrm{ppm}\right): \delta 7.63(\mathrm{~d}, J=8.0 \mathrm{~Hz}, 2 \mathrm{H}, \mathrm{H}-2 "$ \& $\mathrm{H}-$ 6"), 7.31 (d, J = 8.0 Hz, 2H, H-3" \& H-5"), 3.69$3.64\left(\mathrm{~m}, 2 \mathrm{H}, \mathrm{H}_{\mathrm{e}}-2^{\prime} \& \mathrm{H}_{\mathrm{e}}-6^{\prime}\right), 3.20$ (q, J = 7.2 Hz, $\left.2 \mathrm{H}, \mathrm{H}-1^{\prime \prime \prime}\right), 2.87-2.80(\mathrm{~m}, 1 \mathrm{H}, \mathrm{H}-4$ '), 2.60-2.54 (m, $\left.2 \mathrm{H}, \mathrm{H}_{a}-2^{\prime} \& \mathrm{H}_{a}-6^{\prime}\right), 2.42$ (s, 3H, $\left.\mathrm{CH}_{3}-4 "\right)$, 2.12$2.08\left(\mathrm{~m}, 2 \mathrm{H}, \mathrm{H}_{\mathrm{e}}-3^{\prime} \& \mathrm{H}_{\mathrm{e}}-5^{\prime}\right)$, 2.01-1.94 (m, 2H, $\mathrm{H}^{-}$ $\left.3^{\prime} \& \mathrm{H}_{a}-5^{\prime}\right), 1.43\left(\mathrm{t}, \mathrm{J}=7.2 \mathrm{~Hz}, 3 \mathrm{H}, \mathrm{CH}_{3}-2^{\prime \prime \prime}\right)$; EIMS (m/z): $367[\mathrm{M}]^{+}, 266\left[\mathrm{C}_{13} \mathrm{H}_{16} \mathrm{NO}_{3} \mathrm{~S}\right]^{+}, 238$ $\left[\mathrm{C}_{12} \mathrm{H}_{16} \mathrm{NO}_{2} \mathrm{~S}\right]^{+}, \quad 184 \quad\left[\mathrm{C}_{8} \mathrm{H}_{10} \mathrm{NO}_{2} \mathrm{~S}\right]^{+}, \quad 170$ $\left[\mathrm{C}_{7} \mathrm{H}_{8} \mathrm{NO}_{2} \mathrm{~S}\right]^{+}, 155\left[\mathrm{C}_{7} \mathrm{H}_{7} \mathrm{O}_{2} \mathrm{~S}\right]^{+}, \quad 91\left[\mathrm{C}_{7} \mathrm{H}_{7}\right]^{+}, 83$ $\left[\mathrm{C}_{5} \mathrm{H}_{9} \mathrm{~N}^{+}, 29\left[\mathrm{C}_{2} \mathrm{H}_{5}\right]^{+}\right.$.

\section{2-(Propan-1-ylthio)-5-(1-(4-toluenesulfonyl) piperidin-4-yl)-1,3,4-oxadiazole (7b)}

White amorphous solid; Yield: 88 \%; M.P.: 178$180{ }^{\circ} \mathrm{C}$; Mol. formula: $\mathrm{C}_{17} \mathrm{H}_{23} \mathrm{~N}_{3} \mathrm{O}_{3} \mathrm{~S}_{2}$; Mol. weight: 381; IR $\left(\mathrm{KBr}, \mathrm{cm}^{-1}\right)$ : $U_{\max } 3065$ (Aromatic $\mathrm{C}-\mathrm{H}$ ), 1645 (Oxadiazole $\mathrm{C}=\mathrm{N}$ ), 1531 (Aromatic $\mathrm{C}=\mathrm{C}$ ), 1335 (Sulfamoyl S=O), 1249 \& 1079 (Oxadiazole C-O-C); ${ }^{1} \mathrm{H}-\mathrm{NMR}\left(400 \mathrm{MHz}, \mathrm{CDCl}_{3}, \mathrm{ppm}\right): \delta 7.62$ (d, $J=8.0 \mathrm{~Hz}, 2 \mathrm{H}, \mathrm{H}-2 "$ \& H-6"), 7.33 (d, $J=8.0$ $\mathrm{Hz}, 2 \mathrm{H}, \mathrm{H}-3$ " \& H-5"), 3.68-3.65 (m, 2H, $\mathrm{H}_{\mathrm{e}}-2$ ' \& $\left.\mathrm{H}_{\mathrm{e}}-6^{\prime}\right), 3.18$ (t, $\left.\mathrm{J}=6.8 \mathrm{~Hz}, 2 \mathrm{H}, \mathrm{H}-1^{\prime \prime \prime}\right), 2.87-2.80$ (m, 1H, H-4'), 2.58-2.52 (m, 2H, $\left.\mathrm{H}_{a}-2^{\prime} \& \mathrm{H}_{a}-6^{\prime}\right)$, 2.42 (s, 3H, $\left.\mathrm{CH}_{3}-4^{\prime \prime}\right), 2.10-2.06\left(\mathrm{~m}, 2 \mathrm{H}, \mathrm{H}_{e}-3^{\prime}\right.$ \& $\left.\mathrm{H}_{e}-5^{\prime}\right)$, 2.01-1.94 (m, 2H, $\left.\mathrm{H}_{a}-3^{\prime} \& \mathrm{H}_{a}-5^{\prime}\right), 1.52-1.41$ (m, 2H, H-2"'), 0.95 (t, J = 7.2 Hz, 3H, $\left.\mathrm{CH}_{3}-3^{\prime \prime \prime}\right)$; EIMS (m/z): $381\left[\mathrm{M}^{+}, 266\left[\mathrm{C}_{13} \mathrm{H}_{16} \mathrm{NO}_{3} \mathrm{~S}\right]^{+}, 238\right.$ $\left[\mathrm{C}_{12} \mathrm{H}_{16} \mathrm{NO}_{2} \mathrm{~S}\right]^{+}, \quad 184 \quad\left[\mathrm{C}_{8} \mathrm{H}_{10} \mathrm{NO}_{2} \mathrm{~S}\right]^{+}, \quad 170$ $\left[\mathrm{C}_{7} \mathrm{H}_{8} \mathrm{NO}_{2} \mathrm{~S}\right]^{+}, \quad 155\left[\mathrm{C}_{7} \mathrm{H}_{7} \mathrm{O}_{2} \mathrm{~S}\right]^{+}, \quad 91\left[\mathrm{C}_{7} \mathrm{H}_{7}\right]^{+}, 83$ $\left[\mathrm{C}_{5} \mathrm{H}_{9} \mathrm{~N}\right]^{+}, 43\left[\mathrm{C}_{3} \mathrm{H}_{7}\right]^{+}$.

\section{2-(Propan-2-ylthio)-5-(1-(4-toluenesulfonyl) piperidin-4-yl)-1,3,4-oxadiazole (7c)}

Fluffy white amorphous solid; Yield: $78 \%$; M.P.: 116-128 ${ }^{\circ} \mathrm{C}$; Mol. formula: $\mathrm{C}_{17} \mathrm{H}_{23} \mathrm{~N}_{3} \mathrm{O}_{3} \mathrm{~S}_{2}$; Mol. 
weight: 381 ; IR $\left(\mathrm{KBr}, \mathrm{cm}^{-1}\right)$ : $U_{\max } 3065$ (Aromatic $\mathrm{C}-\mathrm{H}$ ), 1651 (Oxadiazole $\mathrm{C}=\mathrm{N}$ ), 1535 (Aromatic $\mathrm{C}=\mathrm{C}$ ), 1336 (Sulfamoyl $\mathrm{S}=\mathrm{O}$ ), 1253 \& 1084 (Oxadiazole C-O-C); ${ }^{1} \mathrm{H}-\mathrm{NMR} \quad(400 \mathrm{MHz}$, $\left.\mathrm{CDCl}_{3}, \mathrm{ppm}\right): \delta 7.61(\mathrm{~d}, J=8.0 \mathrm{~Hz}, 2 \mathrm{H}, \mathrm{H}-2 "$ \& $\mathrm{H}-$ 6"), 7.30 (d, J = 8.0 Hz, 2H, H-3" \& H-5"), 3.70$3.65\left(\mathrm{~m}, 2 \mathrm{H}, \mathrm{H}_{e}-2^{\prime} \& \mathrm{H}_{e}-6^{\prime}\right), 3.10-3.18(\mathrm{~m}, 1 \mathrm{H}, \mathrm{H}-$ 1"'), 2.86-2.79 (m, 1H, H-4'), 2.61-2.55 (m, 2H, $\left.\mathrm{H}_{a}-2^{\prime} \& \mathrm{H}_{a}-6^{\prime}\right), 2.42$ (s, 3H, $\left.\mathrm{CH}_{3}-4^{\prime \prime}\right), 2.11-2.07$ (m, $\left.2 \mathrm{H}, \mathrm{H}_{e}-3^{\prime} \& \mathrm{H}_{e}-5^{\prime}\right), 2.02-1.95\left(\mathrm{~m}, 2 \mathrm{H}, \mathrm{H}_{a}-3^{\prime} \& \mathrm{H}_{a}-\right.$ 5'), 1.48 (d, J = 7.2 Hz, 6H, $\left.\mathrm{CH}_{3}-2^{\prime \prime \prime} \& \mathrm{CH}_{3}-3^{\prime \prime \prime}\right)$; EIMS (m/z): $381[\mathrm{M}]^{+}, 266\left[\mathrm{C}_{13} \mathrm{H}_{16} \mathrm{NO}_{3} \mathrm{~S}\right]^{+}, 238$ $\left[\mathrm{C}_{12} \mathrm{H}_{16} \mathrm{NO}_{2} \mathrm{~S}\right]^{+}, \quad 184 \quad\left[\mathrm{C}_{8} \mathrm{H}_{10} \mathrm{NO}_{2} \mathrm{~S}\right]^{+}, \quad 170$ $\left[\mathrm{C}_{7} \mathrm{H}_{8} \mathrm{NO}_{2} \mathrm{~S}\right]^{+}, 155\left[\mathrm{C}_{7} \mathrm{H}_{7} \mathrm{O}_{2} \mathrm{~S}\right]^{+}, 91\left[\mathrm{C}_{7} \mathrm{H}_{7}\right]^{+}, 83$ $\left[\mathrm{C}_{5} \mathrm{H}_{9} \mathrm{~N}\right]^{+}, 43\left[\mathrm{C}_{3} \mathrm{H}_{7}\right]^{+}$.

\section{2-(Butan-1-ylthio)-5-(1-(4-toluenesulfonyl) piperidin-4-yl)-1,3,4-oxadiazole (7d)}

White crystalline solid; Yield: 75 \%; M.P.: 132$134{ }^{\circ} \mathrm{C}$; Mol. formula: $\mathrm{C}_{18} \mathrm{H}_{25} \mathrm{~N}_{3} \mathrm{O}_{3} \mathrm{~S}_{2}$; Mol. weight: 395; IR $\left(\mathrm{KBr}, \mathrm{cm}^{-1}\right)$ : $U_{\max } 3060$ (Aromatic $\mathrm{C}-\mathrm{H}$ ), 1642 (Oxadiazole $\mathrm{C}=\mathrm{N}$ ), 1528 (Aromatic $\mathrm{C}=\mathrm{C}$ ), 1334 (Sulfamoyl S=O), 1244 \& 1075 (Oxadiazole $\mathrm{C}-\mathrm{O}-\mathrm{C}$ ); ${ }^{1} \mathrm{H}-\mathrm{NMR}\left(400 \mathrm{MHz}, \mathrm{CDCl}_{3}, \mathrm{ppm}\right): \delta 7.60$ (d, $J=8.0 \mathrm{~Hz}, 2 \mathrm{H}, \mathrm{H}-2 "$ \& H-6"), 7.30 (d, $J=8.0$ $\mathrm{Hz}, 2 \mathrm{H}, \mathrm{H}-3$ " \& H-5"), 3.65-3.61 (m, 2H, He $\mathrm{e}_{\mathrm{e}}$ ' \& $\left.\mathrm{H}_{e}-6^{\prime}\right), 3.18\left(\mathrm{t}, J=6.8 \mathrm{~Hz}, 2 \mathrm{H}, \mathrm{H}-1^{\prime \prime \prime}\right), 2.84-2.78$ (m, $\left.1 \mathrm{H}, \mathrm{H}-4^{\prime}\right), 2.56-2.50\left(\mathrm{~m}, 2 \mathrm{H}, \mathrm{H}_{a}-2^{\prime} \& \mathrm{H}_{a}-6^{\prime}\right)$, 2.41 (s, 3H, $\left.\mathrm{CH}_{3}-4^{\prime \prime}\right), 2.12-2.08\left(\mathrm{~m}, 2 \mathrm{H}, \mathrm{H}_{e}-3^{\prime}\right.$ \& $\left.\mathrm{H}_{e}-5^{\prime}\right), 2.01-1.94\left(\mathrm{~m}, 2 \mathrm{H}, \mathrm{H}_{a}-3^{\prime} \& \mathrm{H}_{a}-5^{\prime}\right), 1.36$ (qui, $\left.J=6.8 \mathrm{~Hz}, 2 \mathrm{H}, \mathrm{H}-2^{\prime \prime \prime}\right), 1.15$ (sex, $J=6.8 \mathrm{~Hz}, 2 \mathrm{H}$, $\left.\mathrm{H}-3^{\prime \prime \prime}\right), 0.95$ (t, $\left.J=7.2 \mathrm{~Hz}, 3 \mathrm{H}, \mathrm{CH}_{3}-4{ }^{\prime \prime \prime}\right)$; EIMS $(\mathrm{m} / \mathrm{z}): 395[\mathrm{M}]^{+}, \quad 266 \quad\left[\mathrm{C}_{13} \mathrm{H}_{16} \mathrm{NO}_{3} \mathrm{~S}\right]^{+}, \quad 238$ $\left[\mathrm{C}_{12} \mathrm{H}_{16} \mathrm{NO}_{2} \mathrm{~S}\right]^{+}, \quad 184 \quad\left[\mathrm{C}_{8} \mathrm{H}_{10} \mathrm{NO}_{2} \mathrm{~S}\right]^{+}, \quad 170$ $\left[\mathrm{C}_{7} \mathrm{H}_{8} \mathrm{NO}_{2} \mathrm{~S}\right]^{+}, \quad 155\left[\mathrm{C}_{7} \mathrm{H}_{7} \mathrm{O}_{2} \mathrm{~S}\right]^{+}, 91\left[\mathrm{C}_{7} \mathrm{H}_{7}\right]^{+}, 83$ $\left[\mathrm{C}_{5} \mathrm{H}_{9} \mathrm{~N}\right]^{+}, 57\left[\mathrm{C}_{4} \mathrm{H}_{9}\right]^{+}$.

\section{2-(Butan-2-ylthio)-5-(1-(4- toluenesulfonyl)piperidin-4-yl)-1,3,4- oxadiazole (7e)}

Grayish white amorphous solid; Yield: $74 \%$; M.P.: 125-127 ${ }^{\circ} \mathrm{C}$; Mol. formula: $\mathrm{C}_{18} \mathrm{H}_{25} \mathrm{~N}_{3} \mathrm{O}_{3} \mathrm{~S}_{2}$; Mol. weight: 395; IR $\left(\mathrm{KBr}, \mathrm{cm}^{-1}\right): U_{\max } 3060$ (Aromatic C-H), 1653 (Oxadiazole $\mathrm{C}=\mathrm{N}$ ), 1535 (Aromatic $\mathrm{C}=\mathrm{C}$ ), 1344 (Sulfamoyl $\mathrm{S}=\mathrm{O}$ ), 1252 \& 1083 (Oxadiazole C-O-C); ${ }^{1} \mathrm{H}-\mathrm{NMR}(400 \mathrm{MHz}$, $\left.\mathrm{CDCl}_{3}, \mathrm{ppm}\right): \delta 7.60(\mathrm{~d}, J=8.0 \mathrm{~Hz}, 2 \mathrm{H}, \mathrm{H}-2 "$ \& $\mathrm{H}-$ 6"), 7.30 (d, J = 8.0 Hz, 2H, H-3" \& H-5"), 3.65$3.61\left(\mathrm{~m}, 2 \mathrm{H}, \mathrm{H}_{e}-2^{\prime} \& \mathrm{H}_{e}-6^{\prime}\right), 3.12-3.05(\mathrm{~m}, 1 \mathrm{H}, \mathrm{H}-$ 1"'), 2.84-2.78 (m, 1H, H-4'), 2.56-2.50 (m, 2H, $\left.\mathrm{H}_{a}-2^{\prime} \& \mathrm{H}_{a}-6^{\prime}\right), 2.41$ (s, 3H, $\left.\mathrm{CH}_{3}-4 "\right)$, 2.12-2.08 (m, $\left.2 \mathrm{H}, \mathrm{H}_{e}-3^{\prime} \& \mathrm{H}_{e}-5^{\prime}\right), 2.01-1.94\left(\mathrm{~m}, 2 \mathrm{H}, \mathrm{H}_{a}-3^{\prime} \& \mathrm{H}_{a^{-}}\right.$ $5^{\prime}$ ), 1.98 (qui, $\left.J=6.8 \mathrm{~Hz}, 2 \mathrm{H}, \mathrm{H}-2^{\prime \prime \prime}\right), 1.68$ (d, $J=$ $\left.6.8 \mathrm{~Hz}, 2 \mathrm{H}, \mathrm{CH}_{3}-4{ }^{\prime \prime \prime}\right), 0.95(\mathrm{t}, J=6.8 \mathrm{~Hz}, 3 \mathrm{H}$, $\left.\mathrm{CH}_{3}-3^{\prime \prime \prime}\right) ; \quad$ EIMS $(\mathrm{m} / \mathrm{z}): 395\left[\mathrm{M}^{+}, \quad 266\right.$ $\left[\mathrm{C}_{13} \mathrm{H}_{16} \mathrm{NO}_{3} \mathrm{~S}\right]^{+}, \quad 238\left[\mathrm{C}_{12} \mathrm{H}_{16} \mathrm{NO}_{2} \mathrm{~S}\right]^{+}, \quad 184$ $\left[\mathrm{C}_{8} \mathrm{H}_{10} \mathrm{NO}_{2} \mathrm{~S}\right]^{+}, 170\left[\mathrm{C}_{7} \mathrm{H}_{8} \mathrm{NO}_{2} \mathrm{~S}\right]^{+}, 155\left[\mathrm{C}_{7} \mathrm{H}_{7} \mathrm{O}_{2} \mathrm{~S}\right]^{+}$, $91\left[\mathrm{C}_{7} \mathrm{H}_{7}\right]^{+}, 83\left[\mathrm{C}_{5} \mathrm{H}_{9} \mathrm{~N}\right]^{+}, 57\left[\mathrm{C}_{4} \mathrm{H}_{9}\right]^{+}$.

\section{2-(Pentan-1-ylthio)-5-(1-(4-toluenesulfonyl) piperidin-4-yl)-1,3,4-oxadiazole (7f)}

Fluffy white amorphous solid; Yield: $78 \%$; M.P.: 120-123 ${ }^{\circ} \mathrm{C}$; Mol. formula: $\mathrm{C}_{19} \mathrm{H}_{27} \mathrm{~N}_{3} \mathrm{O}_{3} \mathrm{~S}_{2}$; Mol. weight: 409; IR $\left(\mathrm{KBr}, \mathrm{cm}^{-1}\right)$ : $U_{\max } 3060$ (Aromatic $\mathrm{C}-\mathrm{H}$ ), 1642 (Oxadiazole $\mathrm{C}=\mathrm{N}$ ), 1528 (Aromatic $\mathrm{C}=\mathrm{C}$ ), 1334 (Sulfamoyl $\mathrm{S}=\mathrm{O}$ ), 1244 \& 1075 (Oxadiazole C-O-C); ${ }^{1} \mathrm{H}-\mathrm{NMR}\left(400 \mathrm{MHz} \mathrm{CDCl}_{3}\right.$, ppm): $\delta 7.60$ (d, J = 8.0 Hz, 2H, H-2" \& H-6"), $7.30(\mathrm{~d}, J=8.0 \mathrm{~Hz}, 2 \mathrm{H}, \mathrm{H}-3 "$ \& H-5"), 3.65-3.61 (m, 2H, $\left.\mathrm{H}_{e}-2^{\prime} \& \mathrm{H}_{\mathrm{e}}-6^{\prime}\right), 3.18(\mathrm{t}, \mathrm{J}=6.8 \mathrm{~Hz}, 2 \mathrm{H}, \mathrm{H}-$ $\left.1^{\prime \prime \prime}\right), 2.84-2.78(\mathrm{~m}, 1 \mathrm{H}, \mathrm{H}-4$ '), 2.56-2.50 (m, $2 \mathrm{H}$, $\left.\mathrm{H}_{\mathrm{a}}-2^{\prime} \& \mathrm{H}_{\mathrm{a}}-6^{\prime}\right), 2.41$ (s, $\left.3 \mathrm{H}, \mathrm{CH}_{3}-4^{\prime \prime}\right), 2.12-2.08(\mathrm{~m}$, $\left.2 \mathrm{H}, \mathrm{H}_{e}-3^{\prime} \& \mathrm{H}_{e}-5^{\prime}\right), 2.01-1.94\left(\mathrm{~m}, 2 \mathrm{H}, \mathrm{H}_{a}-3^{\prime} \& \mathrm{H}_{a^{-}}\right.$ $5^{\prime}$ ), 1.36 (qui, $\left.J=6.8 \mathrm{~Hz}, 2 \mathrm{H}, \mathrm{H}-2{ }^{\prime \prime}\right), 1.25-1.19$ (m, 4H, H-3"' \& H-4"'), 0.95 (t, $J=7.2 \mathrm{~Hz}, 3 \mathrm{H}$, $\left.\mathrm{CH}_{3}-5^{\prime \prime \prime}\right) ; \quad$ EIMS $(\mathrm{m} / \mathrm{z}): 409 \quad[\mathrm{M}]^{+}, \quad 266$ $\left[\mathrm{C}_{13} \mathrm{H}_{16} \mathrm{NO}_{3} \mathrm{~S}\right]^{+}, \quad 238 \quad\left[\mathrm{C}_{12} \mathrm{H}_{16} \mathrm{NO}_{2} \mathrm{~S}\right]^{+}, \quad 184$ $\left[\mathrm{C}_{8} \mathrm{H}_{10} \mathrm{NO}_{2} \mathrm{~S}\right]^{+}, 170\left[\mathrm{C}_{7} \mathrm{H}_{8} \mathrm{NO}_{2} \mathrm{~S}\right]^{+}, 155\left[\mathrm{C}_{7} \mathrm{H}_{7} \mathrm{O}_{2} \mathrm{~S}\right]^{+}$, $91\left[\mathrm{C}_{7} \mathrm{H}_{7}\right]^{+}, 83\left[\mathrm{C}_{5} \mathrm{H}_{9} \mathrm{~N}\right]^{+}, 71\left[\mathrm{C}_{5} \mathrm{H}_{11}\right]^{+}$.

\section{2-(Pentan-2-ylthio)-5-(1-(4-toluenesulfonyl) piperidin-4-yl)-1,3,4-oxadiazole (7g)}

Light yellow granular amorphous solid; Yield: 77 $\%$; M.P.: $115-117{ }^{\circ} \mathrm{C}$; Mol. formula: $\mathrm{C}_{19} \mathrm{H}_{27} \mathrm{~N}_{3} \mathrm{O}_{3} \mathrm{~S}_{2}$; Mol. weight: 409; IR $\left(\mathrm{KBr}, \mathrm{cm}^{-1}\right)$ : $U_{\max } 3058$ (Aromatic C-H), 1650 (Oxadiazole $\mathrm{C}=\mathrm{N}$ ), 1531 (Aromatic $\mathrm{C}=\mathrm{C}$ ), 1341 (Sulfamoyl $\mathrm{S}=\mathrm{O}$ ), 1251 \& 1082 (Oxadiazole $\mathrm{C}-\mathrm{O}-\mathrm{C}$ ); ${ }^{1} \mathrm{H}-$ NMR (400 MHz, CDCl $3, \mathrm{ppm}): \delta 7.61(\mathrm{~d}, J=8.0$ $\mathrm{Hz}, 2 \mathrm{H}, \mathrm{H}-2 "$ \& H-6"), 7.32 (d, J = 8.0 Hz, 2H, H3" \& H-5"), 3.63-3.59 (m, 2H, $\left.\mathrm{H}_{e}-2^{\prime} \& \mathrm{H}_{e}-6^{\prime}\right), 3.10-$ $3.04\left(\mathrm{~m}, 1 \mathrm{H}, \mathrm{H}-1^{\prime \prime}\right), 2.84-2.78\left(\mathrm{~m}, 1 \mathrm{H}, \mathrm{H}-4{ }^{\prime}\right)$, 2.56-2.50 (m, 2H, $\left.\mathrm{H}_{a}-2^{\prime} \& \mathrm{H}_{a}-6^{\prime}\right), 2.41(\mathrm{~s}, 3 \mathrm{H}$, $\left.\mathrm{CH}_{3}-4^{\prime \prime}\right), 2.12-2.08\left(\mathrm{~m}, 2 \mathrm{H}, \mathrm{H}_{\mathrm{e}}-3^{\prime} \& \mathrm{H}_{e}-5^{\prime}\right), 2.01-$ $1.94\left(\mathrm{~m}, 2 \mathrm{H}, \mathrm{H}_{\mathrm{a}}-3^{\prime} \& \mathrm{H}_{a}-5^{\prime}\right), 2.05$ (q, $J=6.8 \mathrm{~Hz}$, $\left.2 \mathrm{H}, \mathrm{H}-2{ }^{\prime \prime \prime}\right), 1.73$ (d, J = 7.2 Hz, 2H, $\left.\mathrm{CH}_{3}-5^{\prime \prime \prime}\right), 1.46$ (sex, $\left.J=6.8 \mathrm{~Hz}, 2 \mathrm{H}, \mathrm{CH}_{2}-3{ }^{\prime \prime \prime}\right), 0.97$ (t, $J=7.2 \mathrm{~Hz}$, $\left.3 \mathrm{H}, \quad \mathrm{CH}_{3}-4 " '\right) ; \quad$ EIMS (m/z): $409\left[\mathrm{M}^{+}, 266\right.$ $\left[\mathrm{C}_{13} \mathrm{H}_{16} \mathrm{NO}_{3} \mathrm{~S}\right]^{+}, \quad 238 \quad\left[\mathrm{C}_{12} \mathrm{H}_{16} \mathrm{NO}_{2} \mathrm{~S}\right]^{+}, \quad 184$ $\left[\mathrm{C}_{8} \mathrm{H}_{10} \mathrm{NO}_{2} \mathrm{~S}\right]^{+}, 170\left[\mathrm{C}_{7} \mathrm{H}_{8} \mathrm{NO}_{2} \mathrm{~S}\right]^{+}, 155\left[\mathrm{C}_{7} \mathrm{H}_{7} \mathrm{O}_{2} \mathrm{~S}\right]^{+}$, $91\left[\mathrm{C}_{7} \mathrm{H}_{7}\right]^{+}, 83\left[\mathrm{C}_{5} \mathrm{H}_{9} \mathrm{~N}\right]^{+}, 71\left[\mathrm{C}_{5} \mathrm{H}_{11}\right]^{+}$.

\section{2-(Heptan-1-ylthio)-5-(1-(4-toluenesulfonyl) piperidin-4-yl)-1,3,4-oxadiazole (7h)}

Fluffy white amorphous solid; Yield: $79 \%$; M.P.: 127-129 ${ }^{\circ} \mathrm{C}$; Mol. formula: $\mathrm{C}_{21} \mathrm{H}_{31} \mathrm{~N}_{3} \mathrm{O}_{3} \mathrm{~S}_{2}$; Mol. weight: 437; IR $\left(\mathrm{KBr}, \mathrm{cm}^{-1}\right)$ : $U_{\max } 3055$ (Aromatic $\mathrm{C}-\mathrm{H}$ ), 1655 (Oxadiazole $\mathrm{C}=\mathrm{N}$ ), 1531 (Aromatic $\mathrm{C}=\mathrm{C}$ ), 1335 (Sulfamoyl $\mathrm{S}=\mathrm{O}$ ), 1255 \& 1084

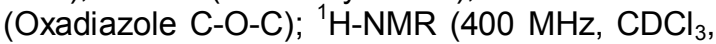
ppm): $\delta 7.62$ (d, J = 8.0 Hz, 2H, H-2" \& H-6"), $7.30(\mathrm{~d}, \mathrm{~J}=8.0 \mathrm{~Hz}, 2 \mathrm{H}, \mathrm{H}-3 "$ \& H-5"), 3.67-3.64 (m, $\left.2 \mathrm{H}, \mathrm{H}_{\mathrm{e}}-2^{\prime} \& \mathrm{H}_{\mathrm{e}}-6^{\prime}\right), 3.17$ (t, J=7.2 Hz, 2H, H$\left.1^{\prime \prime \prime}\right), 2.86-2.81(\mathrm{~m}, 1 \mathrm{H}, \mathrm{H}-4$ '), 2.60-2.54 (m, 2H, $\left.\mathrm{H}_{a}-2^{\prime} \& \mathrm{H}_{a}-6^{\prime}\right), 2.42$ (s, 3H, $\left.\mathrm{CH}_{3}-4^{\prime \prime}\right), 2.11-2.07$ (m, $\left.2 \mathrm{H}, \mathrm{H}_{e}-3^{\prime} \& \mathrm{H}_{e}-5^{\prime}\right), 2.00-1.90\left(\mathrm{~m}, 2 \mathrm{H}, \mathrm{H}_{a}-3^{\prime} \& \mathrm{H}_{a^{-}}\right.$ 
5'), 1.75 (qui, $J=7.2 \mathrm{~Hz}, 2 \mathrm{H}, \mathrm{H}-2$ "'), 1.39 (qui, $J$ $\left.=7.2 \mathrm{~Hz}, 2 \mathrm{H}, \mathrm{H}-3^{\prime \prime \prime}\right), 1.31-1.25(\mathrm{~m}, 6 \mathrm{H}, \mathrm{H}-4$ "' to H-6'"), 0.85 (t, $\left.J=7.2 \mathrm{~Hz}, 3 \mathrm{H}, \mathrm{CH}_{3}-7^{\prime \prime \prime}\right)$; EIMS $(\mathrm{m} / \mathrm{z}): \quad 437 \quad[\mathrm{M}]^{+}, \quad 266 \quad\left[\mathrm{C}_{13} \mathrm{H}_{16} \mathrm{NO}_{3} \mathrm{~S}\right]^{+}, \quad 238$ $\left[\mathrm{C}_{12} \mathrm{H}_{16} \mathrm{NO}_{2} \mathrm{~S}\right]^{+}, \quad 184 \quad\left[\mathrm{C}_{8} \mathrm{H}_{10} \mathrm{NO}_{2} \mathrm{~S}\right]^{+}, \quad 170$ $\left[\mathrm{C}_{7} \mathrm{H}_{8} \mathrm{NO}_{2} \mathrm{~S}\right]^{+}, 155\left[\mathrm{C}_{7} \mathrm{H}_{7} \mathrm{O}_{2} \mathrm{~S}\right]^{+}, 99\left[\mathrm{C}_{7} \mathrm{H}_{15}\right]^{+}, 91$ $\left[\mathrm{C}_{7} \mathrm{H}_{7}\right]^{+}, 83\left[\mathrm{C}_{5} \mathrm{H}_{9} \mathrm{~N}\right]^{+}, 71\left[\mathrm{C}_{5} \mathrm{H}_{11}\right]^{+}, 43\left[\mathrm{C}_{3} \mathrm{H}_{7}\right]^{+}$.

\section{2-(Octan-1-ylthio)-5-(1-(4-toluenesulfonyl) piperidin-4-yl)-1,3,4-oxadiazole (7i)}

Fluffy light yellow amorphous solid; Yield: $75 \%$; M.P.: 130-132 ${ }^{\circ} \mathrm{C}$; Mol. formula: $\mathrm{C}_{22} \mathrm{H}_{33} \mathrm{~N}_{3} \mathrm{O}_{3} \mathrm{~S}_{2}$; Mol. weight: 451 ; IR $\left(\mathrm{KBr}, \mathrm{cm}^{-1}\right): U_{\max } 3050$ (Aromatic C-H), 1651 (Oxadiazole $\mathrm{C}=\mathrm{N}$ ), 1526 (Aromatic $\mathrm{C}=\mathrm{C}$ ), 1332 (Sulfamoyl $\mathrm{S}=\mathrm{O}$ ), 1253 \& 1082 (Oxadiazole C-O-C); ${ }^{1} \mathrm{H}-\mathrm{NMR}(400 \mathrm{MHz}$, $\left.\mathrm{CDCl}_{3}, \mathrm{ppm}\right): \delta 7.61$ (d, J = 8.0 Hz, 2H, H-2" \& H6"), 7.31 (d, J = 8.0 Hz, 2H, H-3" \& H-5"), 3.65$3.62\left(\mathrm{~m}, 2 \mathrm{H}, \mathrm{H}_{e}-2^{\prime} \& \mathrm{H}_{e}-6^{\prime}\right), 3.17(\mathrm{t}, \mathrm{J}=7.2 \mathrm{~Hz}$, $\left.2 \mathrm{H}, \mathrm{H}-1^{\prime \prime \prime}\right), 2.86-2.81\left(\mathrm{~m}, 1 \mathrm{H}, \mathrm{H}-4{ }^{\prime}\right), 2.60-2.54$ (m, $\left.2 \mathrm{H}, \mathrm{H}_{a}-2^{\prime} \& \mathrm{H}_{a}-6^{\prime}\right), 2.42$ (s, 3H, $\left.\mathrm{CH}_{3}-4 "\right), 2.11-$ $2.07\left(\mathrm{~m}, 2 \mathrm{H}, \mathrm{H}_{e}-3^{\prime} \& \mathrm{H}_{\mathrm{e}}-5^{\prime}\right), 2.00-1.90\left(\mathrm{~m}, 2 \mathrm{H}, \mathrm{H}^{-}\right.$ $3^{\prime} \& \mathrm{H}_{a}-5^{\prime}$ ), 1.73 (qui, $\left.J=7.2 \mathrm{~Hz}, 2 \mathrm{H}, \mathrm{H}-2^{\prime \prime \prime}\right), 1.41$ (qui, $\left.J=7.2 \mathrm{~Hz}, 2 \mathrm{H}, \mathrm{H}-3^{\prime \prime \prime}\right), 1.33-1.28(\mathrm{~m}, 6 \mathrm{H}, \mathrm{H}-$ 4"' to H-6"'), 1.12 (sex, $J=7.2 \mathrm{~Hz}, 2 \mathrm{H}, \mathrm{H}-7$ "'), 0.89 (t, J = 7.2 Hz, 3H, $\left.\mathrm{CH}_{3}-8^{\prime \prime \prime}\right)$; $\operatorname{EIMS~(m/z):~} 451$
$[\mathrm{M}]^{+}, 266\left[\mathrm{C}_{13} \mathrm{H}_{16} \mathrm{NO}_{3} \mathrm{~S}\right]^{+}, 238\left[\mathrm{C}_{12} \mathrm{H}_{16} \mathrm{NO}_{2} \mathrm{~S}\right]^{+}, 184$ $\left[\mathrm{C}_{8} \mathrm{H}_{10} \mathrm{NO}_{2} \mathrm{~S}\right]^{+}, 170\left[\mathrm{C}_{7} \mathrm{H}_{8} \mathrm{NO}_{2} \mathrm{~S}\right]^{+}, 155\left[\mathrm{C}_{7} \mathrm{H}_{7} \mathrm{O}_{2} \mathrm{~S}\right]^{+}$, $113\left[\mathrm{C}_{8} \mathrm{H}_{17}\right]^{+}, \quad 91 \quad\left[\mathrm{C}_{7} \mathrm{H}_{7}\right]^{+}, \quad 85 \quad\left[\mathrm{C}_{6} \mathrm{H}_{13}\right]^{+}, 83$ $\left[\mathrm{C}_{5} \mathrm{H}_{9} \mathrm{~N}\right]^{+}, 57\left[\mathrm{C}_{4} \mathrm{H}_{9}\right]^{+}, 29\left[\mathrm{C}_{2} \mathrm{H}_{5}\right]^{+}$.

\section{Antibacterial activity}

The antibacterial activity results are shown in Table 2 and Table 3.

\section{DISCUSSION}

Compound 7a was obtained as fluffy white amorphous solid with molecular formula of $\mathrm{C}_{16} \mathrm{H}_{21} \mathrm{~N}_{3} \mathrm{O}_{3} \mathrm{~S}_{2}$, established through EIMS spectrum. The major absorption bands in IR spectrum appeared at $\mathrm{U}_{\max }\left(\mathrm{cm}^{-1}\right) 3065$ (ar. C-H str.), 1563 (ar. $\mathrm{C}=\mathrm{C}$ str.) \& $1335(\mathrm{~S}=\mathrm{O})$ for sulfamoyl moiety while $1645(\mathrm{C}=\mathrm{N}), 1249 \& 1079$ (C-O-C) confirmed the presence of oxadiazole moiety in the molecule. In EIMS spectrum molecular ion peak appeared at $\mathrm{m} / \mathrm{z} 367$. The other prominent fragments appeared at $\mathrm{m} / \mathrm{z} 266$ for 1-(4-toluenesulfonyl)piperidin-4carbonylcation, at $\mathrm{m} / \mathrm{z} 155$ for 4-toluenesulfonyl cation and at $\mathrm{m} / \mathrm{z} 83$ for piperidine radical cation.

Table 2: Inhibition of antibacterial activity of synthesized compounds

\begin{tabular}{lccccc}
\hline Compound & \multicolumn{5}{c}{ Inhibition (\%) } \\
\cline { 2 - 6 } & $\begin{array}{c}\text { Salmonella } \\
\text { typhi (-) }\end{array}$ & $\begin{array}{c}\text { Escherichia } \\
\text { coli (-) }\end{array}$ & $\begin{array}{c}\text { Pseudomonas } \\
\text { aeruginosa (-) }\end{array}$ & $\begin{array}{c}\text { Staphylococcus } \\
\text { aureus(+) }\end{array}$ & $\begin{array}{c}\text { Bacillus } \\
\text { subtilis (+) }\end{array}$ \\
\hline 5 & $85.68 \pm 1.00$ & $54.90 \pm 0.90$ & $55.21 \pm 0.01$ & $55.52 \pm 0.46$ & $34.77 \pm 0.87$ \\
$7 \mathrm{a}$ & $72.94 \pm 0.35$ & $70.45 \pm 0.58$ & $69.41 \pm 0.10$ & $44.86 \pm 0.60$ & $76.63 \pm 0.43$ \\
$7 \mathrm{~b}$ & $71.02 \pm 0.42$ & $67.25 \pm 0.75$ & $68.54 \pm 0.37$ & $63.61 \pm 0.29$ & $73.10 \pm 0.70$ \\
$7 \mathrm{c}$ & $66.62 \pm 0.76$ & $64.70 \pm 0.20$ & $67.07 \pm 0.05$ & $58.10 \pm 0.45$ & $72.30 \pm 0.83$ \\
$7 \mathrm{~d}$ & $59.14 \pm 1.00$ & $53.75 \pm 0.45$ & $56.13 \pm 0.27$ & $62.90 \pm 0.10$ & $66.73 \pm 0.90$ \\
$7 \mathrm{e}$ & $72.82 \pm 0.64$ & $55.15 \pm 0.67$ & $53.66 \pm 0.73$ & $52.80 \pm 0.90$ & $64.13 \pm 0.47$ \\
$7 \mathrm{f}$ & $73.29 \pm 0.85$ & $55.60 \pm 0.95$ & $51.96 \pm 0.23$ & $56.67 \pm 0.12$ & $62.93 \pm 0.07$ \\
$7 \mathrm{~g}$ & $67.17 \pm 0.41$ & $45.60 \pm 0.90$ & $30.72 \pm 0.67$ & $49.80 \pm 0.43$ & $50.20 \pm 0.53$ \\
$7 \mathrm{~h}$ & $63.84 \pm 0.32$ & $59.95 \pm 0.70$ & $64.46 \pm 0.18$ & $45.88 \pm 0.52$ & $69.63 \pm 0.30$ \\
$7 \mathrm{i}$ & $32.35 \pm 0.35$ & $49.95 \pm 0.05$ & $42.30 \pm 0.42$ & $13.66 \pm 0.10$ & $64.53 \pm 0.33$ \\
\hline Ciprofloxacin & $91.05 \pm 0.68$ & $92.32 \pm 0.42$ & $92.50 \pm 0.34$ & $91.44 \pm 0.64$ & $92.02 \pm 053$ \\
\hline
\end{tabular}

Table 3: MIC of synthesized compounds

\begin{tabular}{lccccc}
\hline Compound & \multicolumn{5}{c}{ MIC $(\mu \mathrm{M})$} \\
\cline { 2 - 6 } & $\begin{array}{c}\text { Salmonella } \\
\text { typhi }(-)\end{array}$ & $\begin{array}{c}\text { Escherichia } \\
\text { coli }(-)\end{array}$ & $\begin{array}{c}\text { Pseudomonas } \\
\text { aeruginosa }(-)\end{array}$ & $\begin{array}{c}\text { Staphylococcus } \\
\text { aureus(+) }\end{array}$ & $\begin{array}{c}\text { Bacillus } \\
\text { subtilis (+) }\end{array}$ \\
\hline 5 & $8.10 \pm 0.41$ & $17.41 \pm 0.18$ & $17.21 \pm 0.23$ & $17.98 \pm 0.51$ & - \\
$7 \mathrm{a}$ & $9.11 \pm 0.40$ & $9.89 \pm 0.45$ & $9.14 \pm 0.72$ & - & $9.11 \pm 0.60$ \\
$7 \mathrm{~b}$ & $9.63 \pm 0.51$ & $10.56 \pm 0.65$ & $10.20 \pm 0.45$ & $12.78 \pm 0.55$ & $9.43 \pm 0.10$ \\
$7 \mathrm{c}$ & $10.34 \pm 0.61$ & $11.21 \pm 0.78$ & $10.16 \pm 0.31$ & $17.20 \pm 0.68$ & $9.87 \pm 0.19$ \\
$7 \mathrm{~d}$ & $14.39 \pm 0.31$ & $17.54 \pm 0.09$ & $17.61 \pm 0.24$ & $14.59 \pm 0.62$ & $10.55 \pm 0.52$ \\
$7 \mathrm{e}$ & $9.22 \pm 0.81$ & $16.43 \pm 0.12$ & $16.54 \pm 0.50$ & $19.28 \pm 0.67$ & $11.46 \pm 0.69$ \\
$7 \mathrm{f}$ & $9.10 \pm 0.10$ & $16.14 \pm 0.65$ & $19.01 \pm 0.14$ & $18.97 \pm 0.38$ & $14.86 \pm 0.15$ \\
$7 \mathrm{~g}$ & $9.89 \pm 0.48$ & - & - & - & $19.70 \pm 0.20$ \\
$7 \mathrm{~h}$ & $12.63 \pm 0.56$ & $15.98 \pm 0.10$ & $11.20 \pm 0.31$ & - & $9.97 \pm 0.09$ \\
$7 \mathrm{i}$ & - & - & - & - & $12.01 \pm 0.87$ \\
\hline Ciprofloxacin & $7.45 \pm 0.58$ & $7.16 \pm 0.58$ & $7.14 \pm 0.18$ & $7.80 \pm 0.19$ & $7.29 \pm 0.90$ \\
\hline
\end{tabular}


The notable fragments have also been sketched in Figure 1. ${ }^{1} \mathrm{H}-\mathrm{NMR}$ spectrum revealed ten resonating peaks nominating the presence of twenty one protons. The aromatic region of ${ }^{1} \mathrm{H}$ NMR spectrum presented two doublets with two proton integration at $\delta 7.63(\mathrm{~J}=8.0 \mathrm{~Hz}, \mathrm{H}-2$ " \& $\mathrm{H}-6 ")$ and 7.31 ( $\mathrm{J}=8.0 \mathrm{~Hz}, \mathrm{H}-3$ " \& $\mathrm{H}-5$ ") for para substituted phenyl ring of 4-toluenesulfony moiety in the molecule. In aliphatic region, four multiplets resonated at $\delta 3.69-3.642 .60-2.54$ 2.12-2.08 and 2.01-1.94 with double integration and one multiplet at $\delta$ 2.87-2.80 with single integration for piperidine moiety. One singlet at $\delta$ 2.42 was assigned to methyl group directly attached to phenyl ring. The S-substituted ethyl group was characterized by two signals, one quartet and one triplet, appearing at $\delta 3.20$ and 1.43 The whole discussion nominated the molecule as 2-ethylthio-5-(1-(4toluenesulfonyl)piperidin-4-yl)-1,3,4-oxadiazole. Likewise the other nominated structures of synthesized molecules were elucidated and sketched in Table 1.

The synthesized compounds were screened against five bacterial strains, including three gram-negative and two gram-positive as discussed in experimental section. The results are shown in table- 2 and table- 3 relative to the reference standard, ciprofloxacin. The MIC values indicated that most of the compounds are active against all strains under consideration. Compound $\mathbf{5}$ showed best activity against $S$. typhi, moderate against others and no activity against $B$. subtilis. This compound executed the MIC of $8.10 \pm 0.41 \mu \mathrm{M}$ relative to that of reference, $7.45 \pm 0.58 \mu \mathrm{M}$ against $S$. typhi bacterial strain. This was the precursor which was further step forwarded into some new molecules to check out their antibacterial potential. Among the substituted compounds, 7af, different compounds showed different antibacterial potential against different bacterial strains. The compound, $\mathbf{7 g}$ possess relatively strong antibacterial potential against $S$. typhi with MIC $9.89 \pm 0.48$ with reference to ciprofloxacin i.e., $7.45 \pm 0.58$. Compound $7 \mathbf{e}-\mathrm{g}$ exhibit good inhibitory potential against $\mathbf{S}$. typhi with MIC values $9.22 \pm 0.81,9.10 \pm 0.10$ and $9.89 \pm 0.48$ respectively as compared to ciprofloxacin i.e., $7.45 \pm 0.58$.

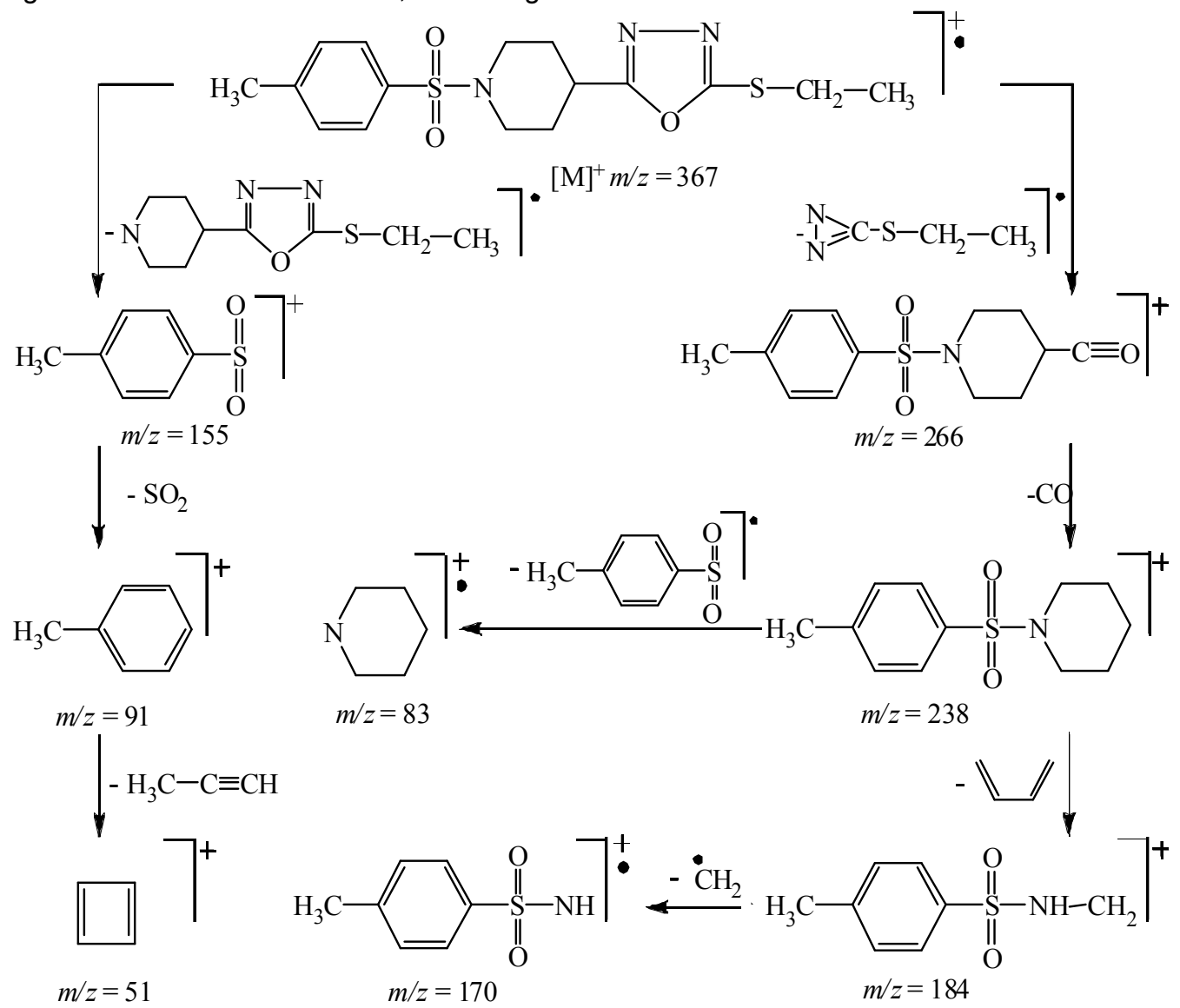

Figure 1: Mass fragmentation pattern of 2-ethylthio-5-(1-(4-toluenesulfonyl)piperidin-4-yl)-1,3,4oxadiazole (7a) 
The molecule, $7 \mathrm{a}$ was the most active against the three strains, with MIC values of $9.11 \pm 0.40$, $9.89 \pm 0.45$ and $9.14 \pm 0.72 \mu \mathrm{M}$ in comparison with ciprofloxacin i.e., $7.45 \pm 0.58,7.16 \pm 0.58$ and $7.14 \pm 0.18 \mu \mathrm{M}$, respectively. This molecule also exhibited the best activity against $B$. subtilis with MIC of $9.11 \pm 0.60 \mu \mathrm{M}$ relative to $7.29 \pm$ $0.90 \mu \mathrm{M}$, the MIC of reference. The best activity of this molecule might be attributed to the presence of ethyl group, the small aliphatic straight chain. The compounds, 7b-f, which remained active against all the strains, might be considered for drug discovery program by the pharmacological industries.

\section{CONCLUSION}

The synthesized molecules have been structurally elucidated by spectroscopic techniques. Molecule $\mathbf{7 a}$ is the strongest antimicrobial agents of all the synthesized compounds. Molecule $\mathbf{5}$ may be further subjected to S-substitution to obtain compounds with comparable or even stronger antimicrobial properties.

\section{ACKNOWLEDGEMENT}

The authors acknowledge Higher Education Commission of Pakistan for financial assistance.

\section{DECLARATIONS}

\section{Conflict of Interest}

No conflict of interest associated with this work.

\section{Contribution of Authors}

The authors declare that this work was done by the authors named in this article and all liabilities pertaining to claims relating to the content of this article will be borne by them.

\section{REFERENCES}

1. Maslat AO, Abussaud M, Tashtoush H, Talib MA. Synthesis, antibacterial, antifungal and genotoxic activity of Bis-1,3,4-oxadiazole derivatives. Pol J Pharmacol 2002; 54: 55-59.

2. Bhardwaj N, Saraf SK, Sharma P, Kumar P. Synthesis, evaluation and characterization of some 1,3,4oxadiazoleas antibacterial agent. Eur J Chem 2009; 6: 1133-1138.

3. Husain A, Ajmal M. Synthesis of novel 1,3,4-oxadiazole derivatives and their biological properties. Acta Pharmaceut 2009; 59: 223-233.
4. Nagaraj, Chaluvaraju KC, Niranjan MS, Kiran S. 1,3,4Oxadiazole: a potent drug candidate with various pharmacological activities. Int J Pharm Pharm Sci 2011; 3: 9-16.

5. Hemavathi SN, Vishu Kumar BK, Lokanatha Rai KM. Synthesis of biological screening of some new 2,5disubstituted 1,3,4-oxadiazoles. Int J Pharm Pharm Sci 2011; 3: 210-214.

6. Cygler M, Schrag JD, Sussman J, Harel LM, Silman I, Gentry MK. Relationship between sequence conservation and three dimensional structure in a large family of esterases, lipases, and related proteins. Protein Sci 1993; 2: 366-382.

7. Omar FA, Mahfouz NM, Rahman MA. Design, synthesis and anti-inflammatory activity of some 1,3,4-oxadiazole derivatives. Eur J Med Chem 1996; 31(10): 819-825.

8. Jin L, Chen J, Song B, Chen Z, Yang S, Li Q, Hu D, Xu $R$. Synthesis, structure, and bioactivity of $N$ '-substituted benzylidene-3,4,5-trimethoxybenzohydrazide and 3acetyl-2-substitutedphenyl-5-(3,4,5-trimethoxyphenyl)2,3-dihydro-1,3,4-oxadiazole derivatives. Bioorg Med Chem Lett 2006; 16(19): 5036-5040.

9. Kumar D, Sundaree S, Johnson EO, Shah K. An efficient synthesis and biological study of novel indolyl-1,3,4oxadiazoles as potent anticancer agents. Bioorg Med Chem Lett 2009; 19(15): 4492-4494.

10. Chin Tan TM, Chen Y, Kong KH, Bai J, Li Y, Lim SG, Ang $T H$, Lam Y. Synthesis and the biological evaluation of 2benzenesulfonylalkyl-5-substituted-sulfanyl-[1,3,4]oxadiazoles as potential anti-hepatitis $B$ virus agents. Antiviral Res 2006; 71(1): 7-14.

11. Yale HL, Losee K. 2-Amino-5-substituted 1,3,4Oxadiazole and 5-Imino-2-substituted $\Delta 2-1,3,4-$ Oxadiazolines. A group of novel muscle relaxants. $J$ Med Chem 1966; 9(4): 478-483.

12. Sanchez-Sancho F, Herrandon B. Short syntheses of (S)pipecolic acid, (R)-coniine, and (S)-coniceine using biocatalytically-generated chiral building block. Tetrahedron: Asymmetry 1998; 9: 1951-1965.

13. Nithiya $S$, Karthik $N$, Jayabharathi J. In vitro antioxidant activity of hindered piperidone derivatives. Int $J$ Pharm Pharm Sci 2011; 3(3): 254-256.

14. Adger B, Dyer U, Hutton G, Woods M. Stereospecific Synthesis of the Anaesthetic Levobupivacaine. Tetrahedron Lett 1996; 37(35): 6399-6402.

15. Aziz-ur-Rehman, Malik A, Riaz N, Nawaz HR, Ahmad H, Nawaz SA, Choudhary MI. Lipoxygenase inhibitory constituents from Periploca aphylla. J Nat Prod 2004; 67: $1450-1454$

16. Aziz-ur-Rehman, Tanveer W, Abbasi MA, Afroz S, Khan KM, Ashraf M, Afzal I. Synthesis, characterization and biological screening of various $\mathrm{N}$-substituted derivatives of sulfonamides. Int J Chem Res 2011; 3(3): 99-104.

17. Kaspady M, Narayanaswamy VK, Raju M, Rao GK. Synthesis, antibacterial activity of 2,4-disubstituted oxazoles and thiazoles as bioesters. Lett Drug Des Discovery 2009; 6: 21-28. 